\title{
Review of: "The role of urban trees in reducing land surface temperatures in European cities"
}

\author{
Bharath Aithal ${ }^{1}$ \\ 1 Indian Institute of Technology Kharagpur
}

Potential competing interests: The author(s) declared that no potential competing interests exist.

The Paper details about the significance urban trees play in reducing the micro-climatic conditions in the form of Land Surface Temperature (LST) for the European cities. The amount of evapotranspiration, albedo and shading were the three most crucial parameters that defines the potential of cooling provided by an urban tree. Shading is directly proportional to the Leaf area index, while transpiration largely varies with the environmental conditions, stomatal behavior, soil moisture limitation and vapour pressure deficit. The study utilised the potential of remote sensing technique for this entire study and considered Landsat data, which provides finest spatial resolution for thermal applications.

LST differences between rural and urban areas were captured and its alteration with LULC types a Generalized Additive Model (GAM) was calibrated for each city. It was observed that the urban trees have lower temperatures than urban fabrics for most of the cities and the cooling provided via trees varies largely with the season (high in summertime and low in winters; vice versa), climatic parameters and geographic conditions. An interesting observation from this communication was that the cooling due to rural forest and urban trees resembled closely with only few notable distinctions. It was found that the urban trees have the capability to cool the urban area with higher potential than treeless green spaces. Interestingly, it was also observed that at some locations urban pastures and green spaces were warmer than the urban fabric.

The absolute temperature difference between urban trees and urban green spaces is generally much lower than the LST difference between the two categories. The analysis based on LST illustrates a clear pattern of cooling provided by different vegetation types. The finding suggests that averaging LST observations might create uncertainties in understanding and planning the cooling potential during the hot extremes. Another factor that can enhance the cooling potential is irrigation, however, it might lead to future water scarcity. Few locations with urban trees were found to provide higher cooling than rural forest, reason being some of the factors such as higher background cooling outweighs the factors that reduces cooling (water stress and insufficient soil volume) to enhance the overall evapotranspiration. For locations with relatively less difference in the cooling from urban trees and rural forests, the evapotranspiration rates can be lower due to increased surface resistance.

Evapotranspiration increased with higher temperatures, higher stomatal conductance. Trees that has the capability to keep their stomata open during hot extremes shows higher cooling effect. Albedo (largely 
depends on incoming shortwave radiation), when compared to evapotranspiration plays a minor role in explaining the differences in the urban trees and urban fabrics over various cities, however, can have significant effect in the dryer region. The cooling potential associated with the tree covered areas, green spaces, rural forests and rural pastures vary substantially throughout the European cities. The reason for this is that rural trees and forest typically exhibit higher surface roughness, thus, increasing the efficiency of heat convention. Trees within street canyons reduces the surface roughness, further leading to reduction in turbulent exchange. Roughness also plays a vital role in explaining UHI magnitude in different region, as it alters the convection of heat - more in wet climate and less in the dry climate.

The article is very well written and excellent contribution to science 